\title{
Identificación de la capsaicina y la deshidrocapsaicina en el extracto de oleorresina obtenido a partir del ají panca (Capsicum chinense)*
}

\author{
Silvana Baldeón Apaestegui** \\ Universidad de Lima. Perú \\ Wilfredo Hernández Gorritti** \\ Instituto de Investigación Científica, Universidad de Lima. Perú \\ Recibido: 20 de junio del 2017 / Aprobado: 13 de julio del 2017
}

\begin{abstract}
RESUMEN: La oleorresina es un extracto líquido que se obtiene a partir de ají y pimientos del género Capsicum, para ser usada frecuentemente en la industria alimentaria. En este trabajo se ha descrito la extracción de la oleorresina a partir del ají panca, por el método Soxhlet. El rendimiento de la oleorresina fue de $22,77 \%$, con el empleo de etanol como solvente extractante. Los capsaicinoides capsaicina y deshidrocapsaicina, presentes en el extracto oleorresina, fueron identificados en etanol a una longitud de onda máxima de $281 \mathrm{~nm}$.
\end{abstract}

Palabras clave: aji panca / oleorresina / estructuras químicas

\section{Identification of capsaicin and dihydrocapsaicin in the oleoresin extract obtained from panca chili (Capsicum chinense)}

Aвstract: Oleoresin is a liquid extract which is obtained from chili and peppers of genus Capsicum, to be used in the food industry very often. This paper describes the oleoresin extraction from aji panca chili pepper by the Soxhlet method. The yield of oleoresin was $22,77 \%$ using ethanol as extraction solvent. The capsaicinoids - capsaicin and dihydrocapsaicinpresent in the oleoresin were identified in ethanol at a maximum $281 \mathrm{~nm}$ wavelength.

Keywords: aji panca chili pepper / oleoresin / chemical structure

* Los autores agradecen a la Facultad de Ingeniería y Arquitectura, y al Instituto de Investigación Científica de la Universidad de Lima por el apoyo brindado para este trabajo. Asimismo, a la Escuela Profesional de Química de la Universidad Nacional Federico Villarreal y al Laboratorio Instrumental de la Sección Química de la PUCP por el apoyo en la caracterización química de la oleorresina.

**Correos electrónicos: silvanabaldeon@gmail.com, whernandez79@yahoo.es 


\section{INTRODUCCIÓN}

El ají panca o Capsicum chinense (figura 1), conocido como ají seco peruano, es una variedad del ají picante (Capsicum baccatum) que se cultiva en la costa del Perú y es el sazonador ideal para la cocina peruana (Plaza y Lock-de-Ugaz, 1997; Salazar, 2016). Este ají -que es valorado tanto por su color como por su sabor - es de apariencia similar al ají amarillo, pero cuando madura adquiere color rojo, carne gruesa, forma cónica y un tamaño que varía entre $8 \mathrm{~cm}$ y $12 \mathrm{~cm}$ de largo y $2,5 \mathrm{~cm}$ y $3 \mathrm{~cm}$ de ancho (Salazar, 2016). El ají panca posee un sabor picante, debido a la presencia de un grupo de compuestos orgánicos denominados capsaicinoides, de los cuales la capsaicina y la deshidrocapsaicina (figura 2) se encuentran en mayor concentración (Restrepo, 2006; Garcés, Arnedo, Abadía, Gil y Álvarez, 2006; Al-Othman, Hadj, Abdelaty y Abdel, 2011; Vásquez et al., 2007). Este ají es rico en vitamina C y ácidos grasos; también contiene algunos carotenoides, como capsantina, capsorrubina, B-caroteno, zeaxantina (figura 3), entre otros componentes que le dan el color característico al ají (Plaza y Lock-de-Ugaz, 1997; Restrepo, 2006; Gonzales, 2010). Por su gran efecto antioxidante, el ají panca se ha convertido en uno de los productos peruanos de mayor consumo; asimismo, es la segunda variedad de ají de mayor cosecha en nuestro país (después del ají amarillo) y es la mejor alternativa para reemplazar los saborizantes y colorantes que existen en el mercado. El ají panca se consume mayormente en los hogares como condimento de alimentos y, además, su uso se ha expandido a la industria cosmética, farmacéutica y alimentaria (Restrepo, 2006; Fernández-Trujillo, 2007).

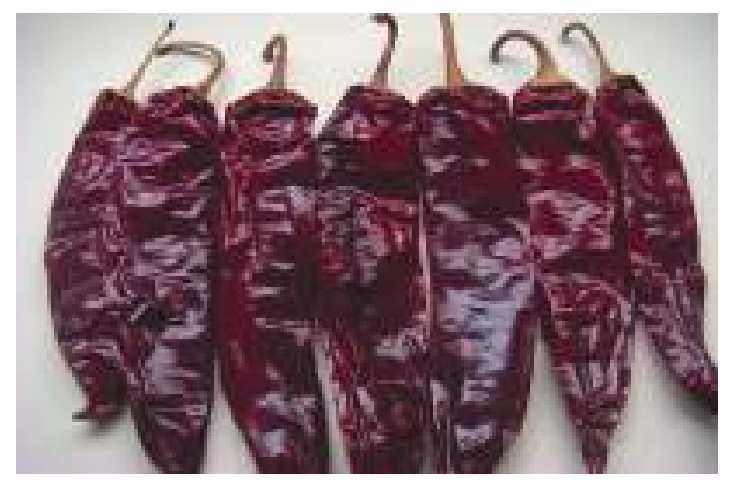

Figura 1. Ají panca (Capsicum chinense) Fotografía: Baldeón y Hernández ${ }^{1}$

1 Todas las fotografías del artículo pertenecen a los autores. 
<smiles>COc1cc(CNC(=O)CCCC/C=C/C(C)C)ccc1O</smiles>

Capsaicina<smiles>COc1cc(CNC(=O)CCCCCCC(C)C)ccc1O</smiles>

\section{Deshidrocapsaicina}

Figura 2. Estructuras químicas de algunos capsaicinoides que se encuentran en mayor concentración en el ají panca Elaboración propia<smiles>CC1=C(/C=C/C(C)=C/C=C/C(C)=C/C=C/C=C(C)/C=C/C=C(C)/C=C/C(=O)C2(C)CC(O)CC2(C)C)C(C)(C)C[C@H](O)C1</smiles><smiles>CC(/C=C/C=C(C)/C=C/C(=O)C1(C)CC(O)CC1(C)C)=C\C=C\C=C(C)\C=C\C=C(/C)C1(C)CC(O)CC1(C)C</smiles><smiles>CC1=C(/C=C/C(C)=C/C=C/C(C)=C/C=C/C=C(C)/C=C/C=C(C)/C=C/C2=C(C)CCCC2(C)C)C(C)(C)CCC1</smiles><smiles>CC1=C(/C=C/C(C)=C/C=C/C(C)=C/C=C/C=C(C)/C=C/C=C(C)/C=C/C2=C(C)CC(O)CC2(C)C)C(C)(C)CC(O)C1</smiles>

Figura 3. Estructuras químicas de algunos carotenoides que se encuentran en el ají panca

Elaboración propia 
El ají panca seco aporta un contenido energético de $292 \mathrm{kcal}$ por cada $100 \mathrm{~g}$ de ají comestible. Su valor nutricional se muestra en la tabla 1.

\begin{tabular}{lr}
\begin{tabular}{l} 
Tabla 1 \\
Composición nutricional proximal por cada \\
100 gramos de ají panca \\
\multicolumn{1}{c}{ Compuesto }
\end{tabular} \\
\hline Proteína & 7 \\
Grasa total & 7,8 \\
Carbohidratos totales & 58,5 \\
Fibra & 22,40 \\
Calcio & 0,142 \\
Hierro & 0,0049 \\
Zinc & 0,00102 \\
Fósforo & 0,209 \\
Vitamina A & 0,001324 \\
Vitamina C & 0,023 \\
Retinol & 0,004412 \\
Riboflavina & 0,00179 \\
Niacina & 0,00355 \\
Tiamina & 0,00013 \\
\hline
\end{tabular}

Fuente: Instituto Nacional de Salud (INS, 2009)

Los frutos del género Capsicum se comercializan de tres formas: en conservas, deshidratadas y como oleorresinas. Uno de los productos de mayor interés que se han obtenido a partir de las especies del género Capsicum es la oleorresina. Esta es una mezcla de derivados capsaicinoides, carotenoides, ácidos grasos y aceites esenciales. La oleorresina es un aceite que se obtiene por extracción con solventes orgánicos polares (acetona y etanol) y no polares (diclorometano, hexano, éter, entre otros), los cuales luego se evaporan para obtener un extracto aceitoso concentrado. La oleorresina es de naturaleza oleosa, viscosa, de color rojo intenso y con un aroma característico, según la procedencia del ají (Salazar, 2016; Fernández-Trujillo, 2007).

La obtención de la oleorresina se justifica por sus excelentes propiedades nutricionales y farmacológicas. Tan es así que su uso como saborizante y colorante para quesos, embutidos, caldos de gallina, salsas, entre otros, se ha visto incrementado en el ámbito nacional e internacional. El 
consumo en el país y las exportaciones de la oleorresina han aumentado en los últimos años, por lo que despierta mucho interés la obtención de este producto (Koo, 2014; Koo, 2017).

En este trabajo se describe la obtención de la oleorresina a partir del ají panca, por medio del método Soxhlet; además, se emplean las técnicas espectroscópicas UV-visible e infrarrojo (FT-IR) para identificar los grupos funcionales y los derivados capsaicinoides capsaicina y deshidrocapsaicina, que están presentes en la oleorresina.

\section{MATERIALES Y EQUIPOS}

El ají panca (216,8 g) empleado en este trabajo se cultivó en la costa del Perú y fue adquirido en los mercados de Lima. Se utilizó un extractor Soxhlet para obtener la oleorresina (extracto líquido) y este extracto fue concentrado en un evaporador rotatorio Heidolph Wmicro, 80 a 200 rpm, 30 a $110{ }^{\circ} \mathrm{C}$. Las propiedades fisicoquímicas de la oleorresina, -como densidad, humedad, índice de acidez y pH-fueron determinadas con un picnómetro de $10 \mathrm{ml}$, una balanza de humedad Sartorius modelo MA30 (40 a $160{ }^{\circ} \mathrm{C}$, precisión $0,01 \mathrm{~g}$ ), un equipo de titulación y un $\mathrm{pH}$-metro digital Mettler Todelo, respectivamente. Los ensayos de solubilidad de la oleorresina se llevaron a cabo empleando los solventes orgánicos metanol, etanol, acetona, diclorometano y cloroformo, de grado reactivo y de marca Sigma-Aldrich.

Los espectros infrarrojos de la oleorresina fueron registrados en un espectrómetro FT-IR modelo Nicolet iS10, con accesorio ATR (attenuated total reflectance) de punta de diamante con single reflection horizontal y 16 barridos; mientras que los espectros UV-visible fueron registrados en un espectrofotómetro UV-visible modelo Thermo Scientific Evolution 201.

\subsection{Procedimiento experimental para la obtención de la oleorresina por el método de extracción Soxhlet (Salazar, 2016)}

En el procedimiento (figura 4), el ají panca se secó en una estufa $\left(\mathrm{T}=60^{\circ} \mathrm{C}\right)$ para eliminar parte de la humedad y luego se trituró para obtener un sólido más pequeño. Cada una de las muestras, previamente pesadas (de 8,21 a 23,35 g), se puso en el cartucho de material poroso y a continuación se introdujo en la cámara de extracción Soxhlet (figura 5). El solvente etanol se colocó en el balón de ebullición que estaba situado debajo de la cámara de extracción. Cuando se evaporó el etanol, el vapor llegó al condensador y se condensó. El etanol líquido cayó sobre el 
cartucho que contenía el ají en polvo y de esta manera se inició la extracción. Cada vez que la cámara se encontraba llena con la mezcla (oleorresina y etanol), regresaba nuevamente al balón a través del sifón lateral (que se hallaba en la parte superior de la cámara). El proceso de extracción, que demoró aproximadamente 6 horas durante 30 ciclos, permitió la concentración de la oleorresina en el balón y la recirculación del solvente. Conforme avanzaba la extracción de la oleorresina, se observaba un color rojizo en el solvente en la cámara de extracción. La extracción finalizó cuando se notó solamente el etanol (incoloro) en la cámara. El extracto de oleorresina frío se filtró y luego se procedió a trasvasar el extracto dentro del balón de un evaporador rotatorio al vacío (figura 6), que operaba a una temperatura de $40{ }^{\circ} \mathrm{C}$ con la finalidad de separar el etanol. La oleorresina concentrada (figura 7) se almacenó en un frasco de color ámbar y se colocó dentro del refrigerador. Finalmente, se desmontó el equipo Soxhlet y el ají húmedo en polvo que se encontraba en el cartucho se llevó a la estufa para proceder a determinar su masa. El rendimiento de la extracción de la oleorresina se calculó dividiendo la masa de la oleorresina, obtenida experimentalmente, entre la masa del ají panca inicial.

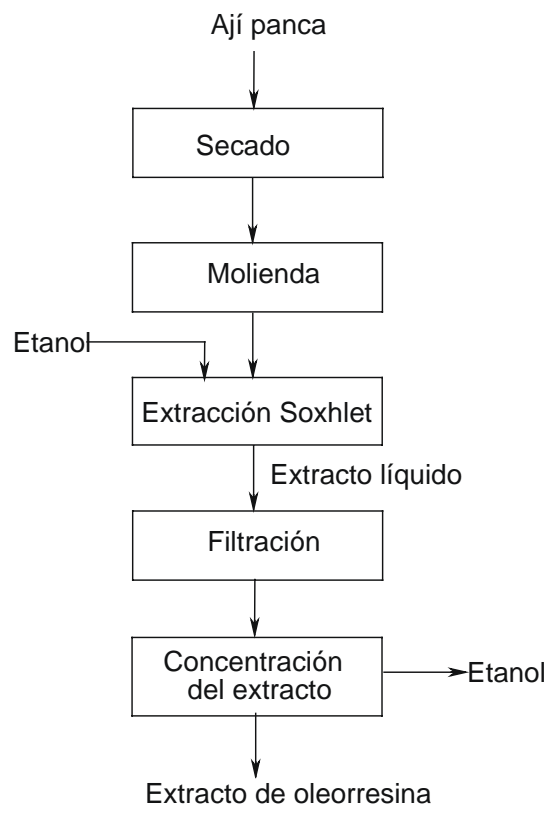

Figura 4. Diagrama de bloques de la obtención de la oleorresina a partir del ají panca

Elaboración propia 


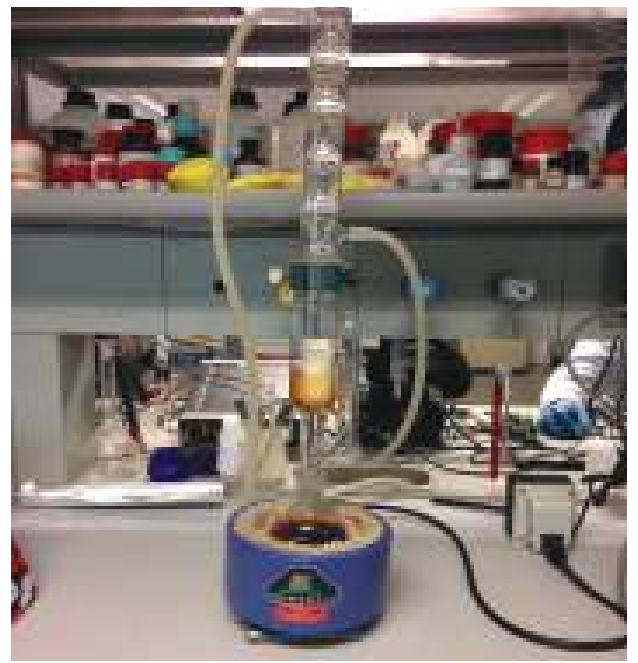

Figura 5. Equipo de extracción Soxhlet

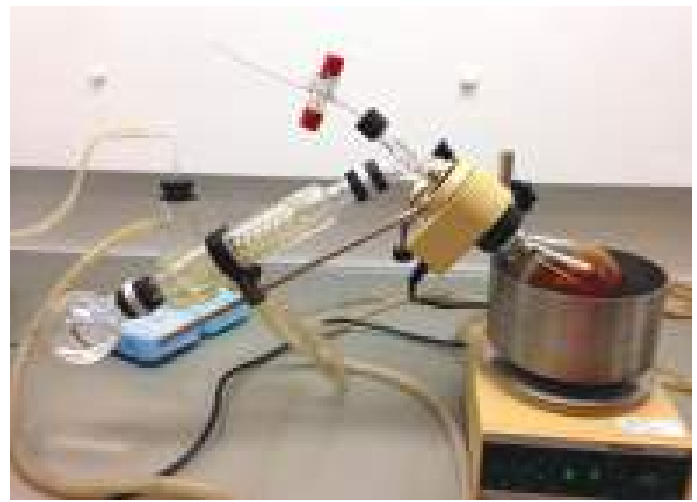

Figura 6. Evaporador rotatorio

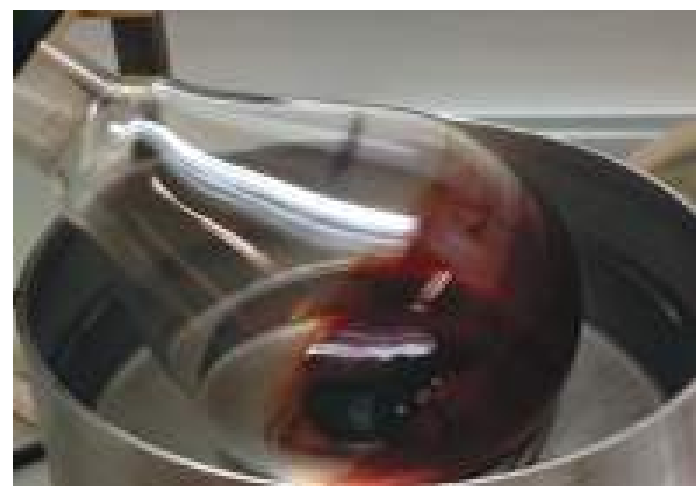

Figura 7. Extracto de la oleorresina luego de separar el etanol 


\section{RESULTADOS}

En el laboratorio de investigación Docimasia, de la Universidad de Lima, se realizó la extracción de la oleorresina a partir del ají panca; asimismo, se determinaron sus propiedades organolépticas (color, aroma y textura) y fisicoquímicas (densidad, humedad, solubilidad, índice de acidez y pH). Por otro lado, se llevó a cabo la caracterización de los grupos funcionales de la oleorresina, mediante espectroscopía infrarroja (FT-IR), y se identificaron los derivados capsaicinoides capsaicina y deshidrocapsaicina, mediante espectroscopía UV-visible.

\subsection{Obtención de la oleorresina}

Se han efectuado nueve ensayos experimentales para la obtención de la oleorresina a partir del ají panca. Los resultados se muestran en la tabla 2 .

Tabla 2

Porcentajes de rendimiento obtenidos para la oleorresina a partir de ají panca

\begin{tabular}{cccccc}
\hline Ensayo & $\begin{array}{c}\text { Masa inicial } \\
\text { del ají panca } \\
\mathbf{( g )}\end{array}$ & $\begin{array}{c}\text { Masa inicial } \\
\text { del ají triturado } \\
\text { que ingresa a } \\
\text { la cámara de } \\
\text { extracción } \\
\mathbf{( g )}\end{array}$ & $\begin{array}{c}\text { Masa final } \\
\text { del ají } \\
\text { luego de la } \\
\text { extracción } \\
\mathbf{( g )}\end{array}$ & $\begin{array}{c}\text { Masa del } \\
\text { extracto } \\
\text { oleorresina } \\
\mathbf{( g )}\end{array}$ & $\begin{array}{c}\text { Porcentaje de } \\
\text { rendimiento }\end{array}$ \\
\hline 1 & 15,26 & 9,88 & 6,49 & 3,39 & 22,21 \\
2 & 13,61 & 8,21 & 5,83 & 2,38 & 17,49 \\
3 & 36,06 & 23,35 & 11,86 & 11,49 & 31,86 \\
4 & 21,24 & 13,75 & 9,44 & 4,31 & 20,29 \\
5 & 20,05 & 12,92 & 9,15 & 3,77 & 18,80 \\
6 & 28,48 & 18,44 & 12,76 & 5,68 & 19,94 \\
7 & 25,84 & 16,73 & 10,82 & 5,91 & 22,87 \\
8 & 30,05 & 19,35 & 12,31 & 7,04 & 23,43 \\
9 & 26,21 & 16,97 & 9,62 & 7,35 & 28,04 \\
\hline & & & & Promedio & 22,77 \\
\cline { 5 - 6 }
\end{tabular}

Elaboración propia

Las propiedades organolépticas y fisicoquímicas halladas en el extracto oleorresina se muestran en la tabla 3. 
Tabla 3

Propiedades organolépticas y fisicoquímicas de la oleorresina

\begin{tabular}{ll}
\hline Color & Rojo \\
Aroma & Característico del ají \\
Textura & Aceitosa \\
Densidad promedio $(\mathbf{g} / \mathbf{m l})$ & 0,97 \\
Humedad $(\%)$ & 24,35 \\
Solubilidad & Soluble en cloroformo y diclorometano. \\
& Parcialmente soluble en metanol, etanol, acetona. \\
Índice de acidez $(\mathbf{m g}$ de $\mathbf{K O H} / \mathbf{g}$ & 39,17 \\
muestra) & \\
$\mathbf{p H}$ & 3,5 \\
\hline
\end{tabular}

Elaboración propia

\subsection{Caracterización de la oleorresina mediante técnicas espectroscópicas FT-IR y UV-visible}

Los espectros infrarrojos fueron registrados en un rango de número de onda desde 500 hasta $4000 \mathrm{~cm}^{-1}$, mientras que los espectros UV-visible fueron obtenidos en un rango de longitud de onda $(\lambda)$ desde 400 hasta $800 \mathrm{~nm}$, tal como se muestra en las figuras 8 y 9 , respectivamente.

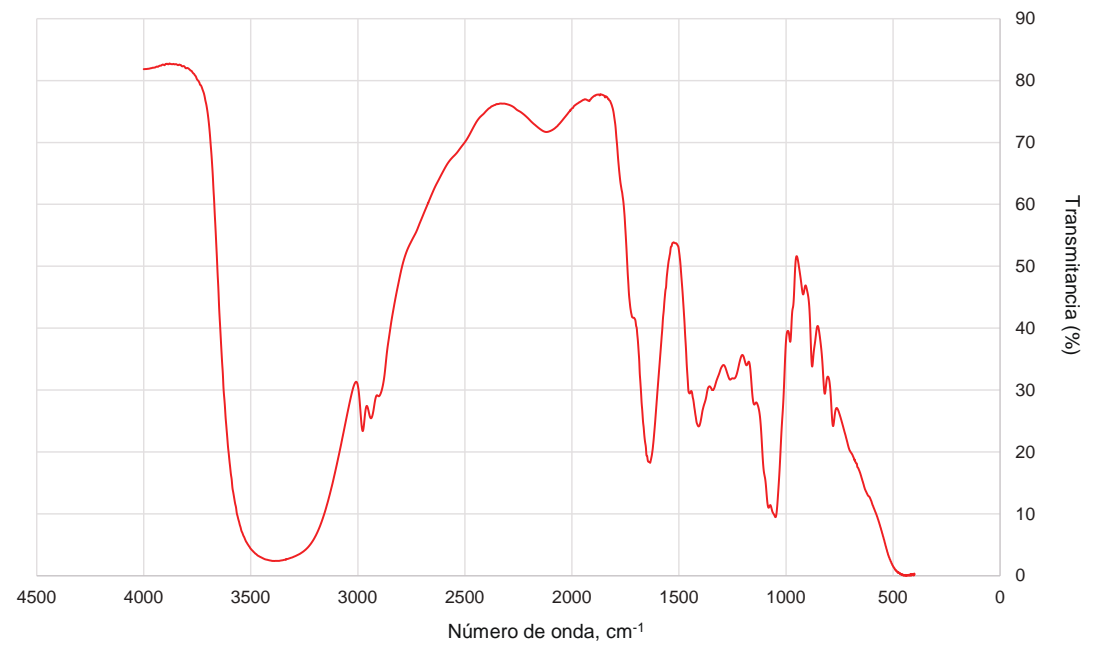

Figura 8. Espectro infrarrojo de la oleorresina Elaboración propia 


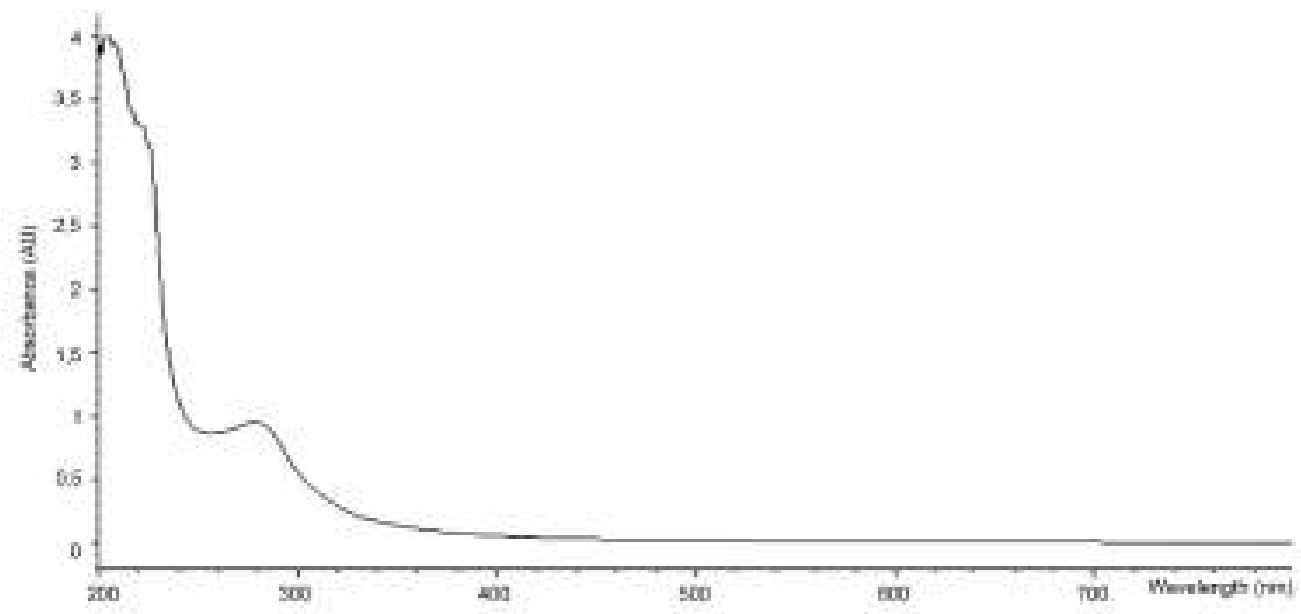

Figura 9. Espectro UV-visible de la oleorresina registrada en diclorometano Nota: Espectro registrado en el espectómetro UV-VIS modelo Agilent 8453 Elaboración propia

\section{DISCUSIÓN}

La obtención de la oleorresina (extracto líquido) a partir del ají panca se llevó a cabo empleando el método Soxhlet y utilizando etanol como solvente extractante. Tal como se muestra en la tabla 2 , se obtuvo un porcentaje promedio de rendimiento de oleorresina del $22,77 \%$ para un ciclo de operación de 5,5 horas. Este porcentaje de rendimiento es mayor que el de los porcentajes de extracción de otras oleorresinas extraídas (de 4,2 \% a 7,7 \%) con etanol a partir de otras variedades de ajíes (Capsicum chinense) y en las que se empleó un tiempo de extracción de 12 horas (Rodríguez, Arango y Urrego, 2000). Asimismo, el porcentaje de rendimiento de oleorresina obtenido en este trabajo fue superior a los rendimientos de extracción alcanzados para otras oleorresinas extraídas del pimentón (de $2,9 \%$ a 4,2 \%), cuyos extractos se obtuvieron utilizando solventes no polares, tales como acetato de etilo y hexano (Salazar, 2016; Fernández-Trujillo, 2007; Cardona et al., 2006). Estos resultados evidencian que el carácter polar del etanol juega un rol importante en la extracción de la oleorresina. 
Las propiedades organolépticas y fisicoquímicas que se encontraron en la oleorresina a partir del ají panca se muestran en la tabla 3. El color, aroma y textura son características típicas de la oleorresina. La densidad de la oleorresina obtenida $(0,97 \mathrm{~g} / \mathrm{ml})$ estuvo dentro del rango esperado de 0,93-0,99 g/ml (Plaza y Lock-de-Ugaz, 1997; Salamanca y Sánchez, 2009). El índice de acidez fue 39,17 mg KOH/g de muestra, un resultado que indica la presencia de ácidos grasos libres en la oleorresina. Cabe agregar que el índice de acidez obtenido en este trabajo fue ligeramente superior con respecto a los índices de acidez informados (11,40-23,44 mg KOH/g de muestra) para otras oleorresinas extraídas (Plaza y Lock-de-Ugaz, 1997).

La caracterización de los grupos funcionales y los componentes de la oleorresina fue llevada a cabo mediante técnicas espectroscópicas de ultravioleta-visible (UV-VIS) e infrarrojo (FT-IR).

Tal como se observa, en el espectro infrarrojo de la oleorresina (figura 8) una banda intensa y ancha aparece a $3387 \mathrm{~cm}^{-1}$, debido al grupo amida (-NHCO-) que compone los derivados capsaicinoides, capsaicina y deshidrocapsaicina. La vibración de estiramiento del grupo -OH está comprendida en la banda ancha debido a que este grupo funcional absorbe en el rango de 3200-3600 $\mathrm{cm}^{-1}$. A $2978 \mathrm{~cm}^{-1}$ se observa una banda débil asignable a la vibración de estiramiento del grupo $-\mathrm{CH}_{2}$ de la cadena alifática (Lotfy y Fawzy, 2014). Los grupos carbonilo $(-\mathrm{C}=\mathrm{O})$ y enlaces dobles $(-\mathrm{C}=\mathrm{C}-)$ se aprecian como una banda intensa a $1635 \mathrm{~cm}^{-1}$, mientras que a $1409 \mathrm{~cm}^{-1}$ surge una banda débil debido a los movimientos de flexiones en el plano del grupo $-\mathrm{CH}_{2}$. Una banda de absorción intensa se manifiesta a $1051 \mathrm{~cm}^{-1}$ debido a las vibraciones de estiramiento de los grupos C-C, C-N y C-O (Fernández, 2007; Baranska y Schulz, 2009). Estos resultados evidencian la presencia de los grupos funcionales orgánicos amida $(-\mathrm{NHCO}-)$, hidroxilo $(-\mathrm{OH})$, alcanos $(-\mathrm{C}-\mathrm{C}-)$ y alquenos $(-\mathrm{C}=\mathrm{C}-)$ en los derivados capsaicina $\mathrm{y}$ deshidrocapsaicina de la oleorresina.

La figura 9 muestra el espectro de absorción UV-visible de la oleorresina registrada en diclorometano. En el espectro aparece una banda de absorción con una longitud de onda máxima de $279 \mathrm{~nm}$, debido a la presencia de las transiciones electrónicas $\mathrm{n} \rightarrow \pi^{*}$ del grupo amida (-NH-CO-) que se encuentra en la capsaicina y deshidrocapsaicina. Este valor obtenido es similar a las longitudes de onda máximas encontradas para los derivados capsaicinoides a partir de otros tipos de 
pimientos (Garcés et al., 2006; Al-Othman et al., 2011; González et al., 2015; Koleva, Mitrev, Maksimova y Spasov, 2013; Fernández, 2007). Las bandas de absorción de los demás constituyentes orgánicos (carotenoides y aceites esenciales, entre otros) de la oleorresina no fueron visualizadas en el rango 400-500 nm del espectro UV-visible. Probablemente, en la longitud de onda máxima de $279 \mathrm{~nm}$ registrada en diclorometano exista una mayor concentración de capsaicinoides, principalmente, capsaicina y deshidrocapsaicina (Liljana, Viktorija, Marija, Rubin y Emilija, 2013; Simonyi, Bikádi, Zsila y Deli, 2003; Schweiggert, Kammerer, Carle y Schieber, 2005). Una caracterización química adicional a la obtenida con la espectrofotometría UV-visible e infrarrojo - con la finalidad de elucidar las fórmulas estructurales de la capsaicina y la deshidrocapsaicina- se da empleando otras técnicas espectroscópicas, tales como la espectrometría de masas y la resonancia magnética nuclear $\left({ }^{1} \mathrm{H},{ }^{13} \mathrm{C}\right)$.

Los resultados de este trabajo son muy alentadores para los investigadores en el área de la química de los alimentos, porque motivan a continuar con futuras indagaciones relacionadas con la separación de los componentes orgánicos que constituyen la oleorresina (como la capsaicina y la deshidrocapsaicina), debido a que estos derivados capsaicinoides cuentan con excelentes propiedades farmacológicas (Chinn, SharmaShivappa y Cotter, 2011; Dong et al., 2014; Giuffrida et al., 2013).

\section{CONCLUSIONES}

- Se ha obtenido oleorresina a partir del ají panca, mediante la técnica Soxhlet, con un buen porcentaje de extracción del 22,77 \%, empleando etanol como solvente extractante.

- Los componentes orgánicos capsaicina y deshidrocapsaicina, presentes en la oleorresina, fueron identificados mediante espectrometría UV-visible, debido a la presencia de una banda de absorción, observada a una longitud de onda máxima de $281 \mathrm{~nm}$, registrada en etanol.

- Los grupos funcionales orgánicos que componen la oleorresina fueron caracterizados mediante la técnica espectroscópica de infrarrojo.

\section{REFERENCIAS}

Al-Othman, Z., Hadj, Y., Abdelaty, M., y Abdel, A. (2011). Determination of Capsaicin and Dihydrocapsaicin in Capsicum Fruit Samples using High Performance Liquid Chromatography. Molecules, 16(10), 8919-8929. 
Baranska, M., y Schulz, H. (2009). Chapter 4. Determination of Alkaloids Through Infrared and Raman Spectroscopy. The Alkaloids: Chemistry and Biology, 67, 217-255.

Cardona, J., Lopera, G., Montoya R., A., Montoya V., A., Peña, J., Gil, M., Ríos, L. y Restrepo, G. (2006). Obtención de oleorresina de pimentón (Capsicum annuum l.). Vitae. Revista de la Facultad de Ciencias Farmacéuticas y Alimentarias, 13(1), 5-9.

Chinn, M., Sharma-Shivappa, R., y Cotter, J. (2011). Solvent Extraction and Quantification of Capsaicinoids from Capsicum chinense. Food and Bioproducts Processing, 89(4), 340-345.

Dong, X., Li, X., Ding, L., Cui, F., Tang, Z., y Liu, Z. (2014). Stage Extraction of Capsaicinoids and Red Pigments from Fresh Red Pepper (Capsicum) Fruits with Ethanol as Solvent. LWT - Food Science and Technology, 59(1), 396-402.

Fernández, G. (2007). Extracción, análisis, estabilidad y síntesis de capsaicinoides (tesis doctoral). Universidad de Cádiz. España.

Fernández-Trujillo, J. (2007). Extracción convencional de oleorresina de pimentón dulce y picante I. Generalidades, composición, proceso e innovaciones y aplicaciones. Grasas y Aceites, 58(3), 252-263.

Garcés, A., Arnedo, M., Abadía, J., Gil, R., y Álvarez, A. (2006). Determination of Capsaicin and Dihydrocapsaicin in Capsicum Fruits by Liquid Chromatography-Electrospray/Time-of-Flight Mass Spectrometry. Journal of Agricultural and Food Chemistry, (54), 9303-9311.

Giuffrida, D., Dugo, P., Torre, G., Bignardi, C., Cavazza, A., Corradini, C., y Dugo, G. (2013). Characterization of 12 capsicum varieties by Evaluation of Their Carotenoid Profile and Pungency Determination, Food Chemistry, 140(4), 794-802.

Gonzales, I. (2010). Caracterización química del color de diferentes variedades de guayaba (Psidium guajava L.) colombiana (tesis de maestría). Universidad Nacional de Colombia, Bogotá.

González, A., Sierra, E., Pérez, R., Vázquez, C., Gallegos, M., López, J., y García, J. (2015). Measurement of Capsaicinoids in Chiltepin Hot Pepper: A Comparison Study Between Spectrophotometric Method and High Performance Liquid Chromatography Analysis. Journal of Chemistry, 1-10. 
Instituto Nacional de Salud. (2009). Tablas peruanas de composición de alimentos (8. ${ }^{\mathrm{a}}$ ed.) Lima: Ministerio de Salud.

Koleva, L., Mitrev, S., Maksimova, V., y Spasov, D. (2013). Content of Capsaicin Extracted from Hot Pepper (Capsicum annuum ssp. microcarpum L.) and its use as an Ecopesticide. Hemijska Industrija, 67(4), 671-675.

Koo, W. (22 de mayo de 2014). Paprika Perú exportación abril 2014 [mensaje en un blog]. Recuperado de http://www.agrodataperu. com/2014/05/paprika-peru-exportacion-abril-2014.html

Koo, W. (25 de abril de 2017). Conservas pimiento, ají paprika, frejol de palo Perú exportación 2017 marzo [mensaje en un blog]. Recuperado de https://www.agrodataperu.com/2017/04/conservas-pimiento-ajipaprika-frejol-palo-peru-exportacion-2017-marzo.html

Liljana, K., Viktorija, M., Marija, S., Rubin, G., y Emilija, I. (2013). The Effect of Different Methods of Extractions of Capsaicin on its Content in the Capsicum Oleoresins. Scientific Works: Food Science, Engineering and Technology, (60), 917-922.

Lotfy, S., y Fawzy, Y. (2014). Characterization and Enhancement of the Electrical Performance of Radiation Modified Poly (Vinyl) Alcohol/Gelatin Copolymer Films Doped with Carotene. Journal of Radiation Research and Applied Sciences, (7), 338-345.

Plaza, A., y Lock-de-Ugaz, O. (1997). Colorantes naturales y la oleorresina de páprika. Revista de Química, 11(1), 73-93.

Restrepo, M. (2006). Oleorresinas de Capsicum en la industria alimentaria. Revista Lasallista de Investigación, 3(2), 43-47.

Rodríguez, L., Arango, J., y Urrego, F. (2000). Obtención de oleorresinas a partir de 3 especies de Capsicum sp. cultivadas en Colombia (Capsicum annuum, Capsicum frutescens, Capscicum chinense). Recuperado de http://avalon.utadeo.edu.co/dependencias/publicaciones/alimentica3/oleorresinas.pdf

Salamanca, M., y Sánchez, M. (2009). Extracción y caracterización de la oleorresina del orégano (Origanum vulgare) (tesis para optar el título de tecnólogo químico). Universidad Tecnológica de Pereira, Colombia.

Salazar, E. (2016). Efecto bacteriostático y bactericida de extractos de aji panca (Capsicum chinense) y pimiento (Capsicum annuum 
var. annuum) sobre cultivos de Escherichia coli ATCC 25922 y Staphylococcus aureus ATCC 25923 (tesis para optar por el título de médico veterinario). Universidad Nacional Mayor de San Marcos.

Schweiggert, U., Kammerer, D., Carle, R., y Schieber, A. (2005). Characterization of Carotenoids and Carotenoid Esters in Red Pepper Pods (Capsicum annuum L.) by High-Performance Liquid Chromatography/Atmospheric Pressure Chemical Ionization Mass Spectrometry. Rapid Communications in Mass Spectrometry, (19), 2617-2628.

Simonyi, M., Bikádi, Z., Zsila, F., y Deli, J. (2003). Supramolecular Exciton Chirality of Carotenoid Aggregates. Chirality, 15(8), 680-698.

Vásquez, F., Miranda, M., Monforte, M., Gutiérrez, G., Velásquez, C., y Nieto, Y. (2007). La biosíntesis de capsaicinoides, el principio picante del chile. Revista Fitotecnia Mexicana, 30(4), 353-360. 
